\title{
OpenSpace: Bringing NASA Missions to the Public
}

Alexander Bock, Charles Hansen and Anders Ynnerman

The self-archived postprint version of this journal article is available at Linköping University Institutional Repository (DiVA):

http:// urn.kb.se/ resolve?urn=urn:nbn:se:liu:diva-152095

N.B.: When citing this work, cite the original publication.

Bock, A., Hansen, C., Ynnerman, A., (2018), OpenSpace: Bringing NASA Missions to the Public, IEEE Computer Graphics and Applications, 38(5), 112-118. https:// doi.org/ 10.1109/MCG.2018.053491735

Original publication available at:

https:// doi.org/ 10.1109/MCG.2018.053491735

Copyright: Institute of Electrical and Electronics Engineers (IEEE)

http:// www.ieee.org/ index.html

(C)2018 IEEE. Personal use of this material is permitted. However, permission to reprint/republish this material for advertising or promotional purposes or for creating new collective works for resale or redistribution to servers or lists, or to reuse any copyrighted component of this work in other works must be obtained from the IEEE. 


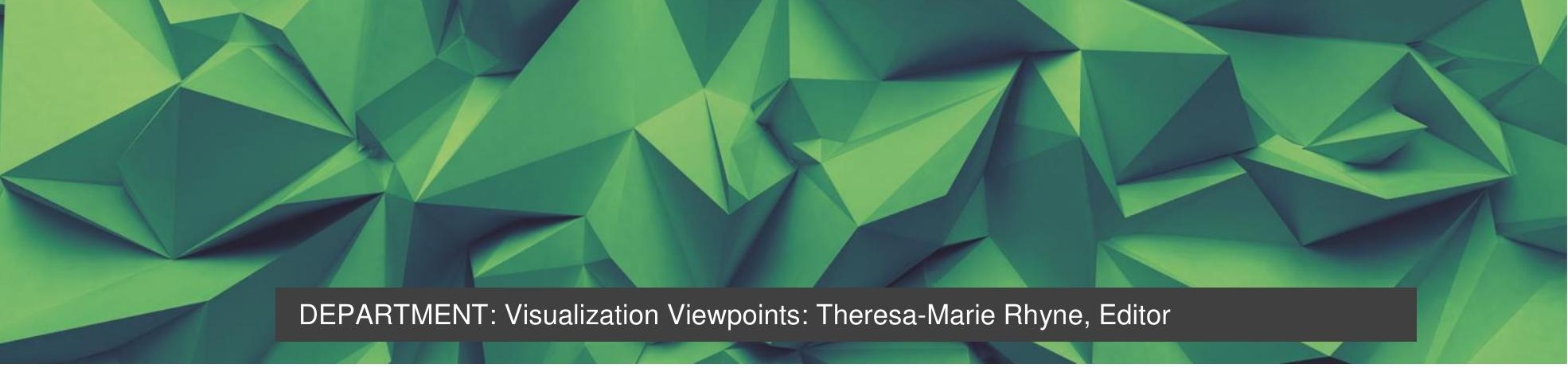

\section{OpenSpace: Bringing NASA Missions to the} Public

Alexander Bock, New York University

Charles Hansen, University of Utah

Anders Ynnerman, Linköping University
The need to communicate and demonstrate the endeavors of space missions has typically been done through produced movies or animations. NASA missions, such as OSIRIS-REx, are the product of years of planning and scientific research. Explaining missions to the public in an immersive setting is challenging and has only previously been possible with produced shows for planetariums or interactively with limited details on the missions and external data ${ }^{1,2}$. Our goal is to provide an interactive experience in which the public can see and experience space missions to better understand the science, the benefit to mankind, and

the challenges faced when conducting deep-space missions.

To achieve this, we have developed the open source interactive data visualization software OpenSpace ${ }^{10}$ designed to visualize the entire known universe and portray our ongoing efforts to investigate the cosmos through large-scale, contextualized, multi-modal astrovisualization. OpenSpace incorporates the latest techniques from visualization research and supports interactive presentation of dynamic data from observations, simulations, and space mission planning and operations $\mathrm{s}^{3,4,5}$. OpenSpace is capable of providing an immersive experience to multiple observers by leveraging the projection capabilities of modern planetariums ${ }^{6}$. This immersive experience will help better inform and educate the general public by allowing interactive exploration of NASA missions. OpenSpace has been developed with partners from the American Museum of Natural History (AMNH), Linköping University (LiU), the Scientific Computing and Imaging Institute (SCI) at the University of Utah, and New York University's Tandon School of Engineering.

\section{SPICE FILES AND NASA MISSIONS}

OpenSpace relies on one continuous coordinate system that enables developers to position and orient data with extreme accuracy. NASA's Spacecraft Planet Instrument Cmatrix Events (SPICE) observation geometry system for planetary science missions, for example, provides us with the ability to display mission planning, data acquisition, and post-mission data analysis using high-resolution data files that are called kernels in SPICE. Each mission has a different set of SPICE kernels that describe the spatial and temporal aspects of the mission and its components. For example, in collaboration with the Applied Physics Laboratory (APL), the OpenSpace team worked to visualize the Navigation and Ancillary Information Facility's (NAIF) SPICEspecified instrument targeting during New Horizon's fly-by of Pluto as designed by the mission science team $^{3}$. SPICE guided visualization of planet and spacecraft orbits, instrument view frustums, and sequenced 
imagery over time allow for comparison of observed data with model output. While New Horizons was a specific application, the techniques apply generically across all space missions with available SPICE data. SPICE compliance accurately defines positions, orbits, trajectories, orientations, and instrument views used in data collection for Earth and planetary science, heliophysics, and astrophysics.

The ability to read SPICE kernels, during mission planning and flight, including actual configurations returned by telemetry during flight, will allow for both historical reconstruction and conceptual visualization of missions within OpenSpace. To date, such full SPICE visualization has only been available in specialized mission planning software, in produced videos such as created by NASA's Science Visualization Studio, or in a limited capability with commercial software ${ }^{2}$. Never before has there been an interactive, deep level of SPICE capability in planetariums or digital theaters using freely available opensource software. Visualizing spacecraft attitude with respect to observation schedule is an important link between mission operations and the science it is designed to acquire. Visualizing the engineering of how space mission science is conducted is especially valuable with respect to public education.

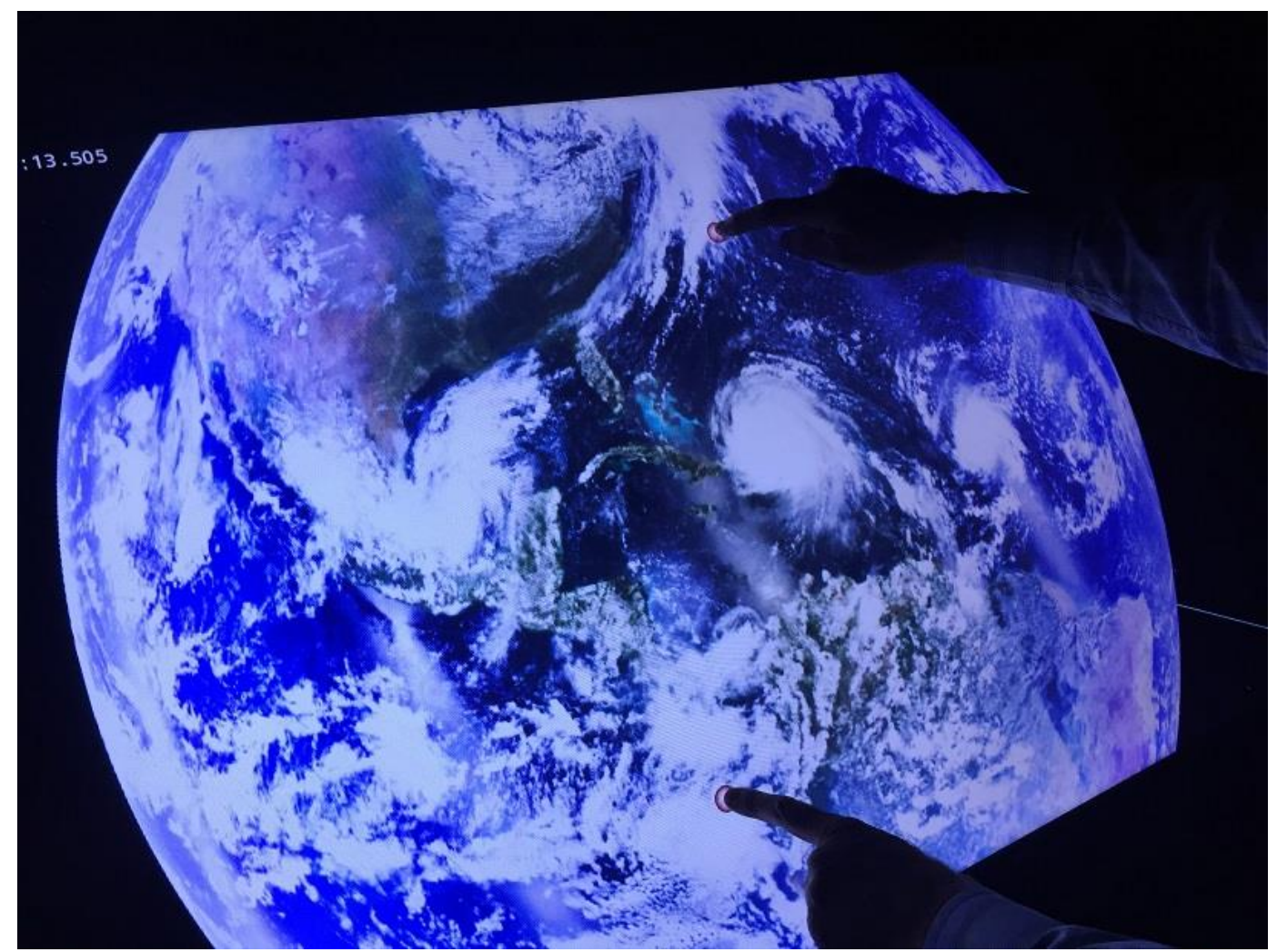

Figure 1. September 9, 2017. Hurricanes West to East (left to right): Katia, Irma, Jose.

\section{DATA SOURCES}

A major product of many space missions are comprehensive planetary maps of the bodies in our solar system. Examples of this include the MESSENGER mission that mapped Mercury, the Lunar Reconnaissance Orbiter at the Moon, the Mars Reconnaissance Orbiter retrieving unprecedented details of the surface of Mars, and the numerous Earth-orbiting spacecraft providing time resolved imagery of our own planet. Many of these maps are provided as images online through the Web Map Service (WMS) standard. Through this standard, images are stored in a tree with varying resolution and only images of appropriate resolution are streamed from an online server to the client application. Streaming data from a variety of online sources enables the rapid exploration of datasets from different spacecraft and also provides the ability to access near-real time 
imagery without the need to download entire image catalogs, thus reducing the latency between data acquisition and visualization. NASA's Global Imagery Browse Services (GIBS) provides daily updates for many of their image sources, such as Suomi-NPP's VIIRS instrument, which provides a full global view of Earth every day depicting weather and cloud cover. Figure 1 shows an image of the three major hurricanes that impacted the United States in 2017 and that was generated from the GIBS dataset and is being inspected by a user in real-time. In addition to visible light measurements, many other mission instruments are available through these protocols, for example cloud layers, ozone concentration, or temperature measurements, thus providing an immense wealth of information that can be dissected in the context of the observing spacecraft.

\section{MULTI-USER IMMERSIVE ENVIRONMENTS}

The benefits of multi-user immersive environments are deeply rooted in the human desire to share experiences and the need for a social context in which knowledge can be put. Immersive multi-user environments come in many different flavors with different advantages and disadvantages. The available systems range from shared VR environments using multiple head mounted displays to immersive environments such as CAVE(s) and large scale display systems such as dome theaters. One key difference between these environments is the number of users who are tracked to provide correct first person view of the virtual world displayed. In multi-user head mounted systems all users are tracked, whereas in CAVE(s) only one person has the "correct" view, and in domes usually no user is tracked.

Our experience shows that multi-user immersion can be facilitated in several different ways without the need for tracking of users. In dome theaters the size of the display and the distance to the users make it possible to generate reasonably correct views for most of the users, and the size of the environments adds the advantage of scalability in number of simultaneous users. The same argument also holds for large flat displays, such as projection walls, that support high resolution immersive experiences as shown in Figure 2. In these environments user interaction is, however, often limited to one user presenting and interacting. In dome theaters this leads to the notion of a mediated immersive experience in which a presenter, often together with a pilot, guides the users (audience) through a demonstration. This as such becomes a powerful tool for immersion, as the story, and the story teller, becomes an integrated component of the immersion. OpenSpace was developed primarily for dome theater immersion. It benefits greatly from the interactive capabilities of the system whereby the multi-users perceive the $3 \mathrm{D}$ effect of the cosmos as long as the projected image reflects camera motion. If it stops, the users suddenly experience a $2 \mathrm{D}$ image projected onto the dome. The $3 \mathrm{D}$ illusion makes this environment extremely compelling for contextualized astrovisualization.

Interaction in itself can also provide a path to immersion. Multi-user interaction on touch tables is today common and enables immersion through shared input and involvement in the content displayed among the users who share interaction, which can sometimes be in conflict, but leading to discussion and thus engagement of users.

\section{TOUCH-TABLE AND PROJECTION WALL}

It has been shown that using large tangible touch surfaces with a multi-touch navigation interface is more engaging to users than mouse and keyboard as well as enhances understanding of navigation control, thus decreasing the learning time of the systems user interface ${ }^{7}$. Additionally, combining a multi-touch interaction model together with a screen-space direct-manipulation formulation produces a user-friendly interface. We have integrated the OpenSpace user interface with a commercial multi-touch display table provided by the company Interspectral $\mathrm{AB}^{8}$.

Astronomical visualizations have long been an interesting application for touch-based interaction. However, due to the scale of the solar system compared to its celestial bodies, any existing object rapidly becomes exceedingly small. A direct-manipulation solution alone becomes non-trivial in an application like this since its formulation require $3 \mathrm{D}$ points in the scene to constrain the manipulation and such points cannot be well tracked in empty space.

The multi-touch interface is implemented using the TUIO library to support a variety of multi-touch devices, although we primarily targeted the multi-touch table. Using this interface, the user can interact with any ce- 
lestial body in the scene and traverse in an expected way through the multi-touch gestures. For example, Figure 1 shows interaction with satellite imagery weather data. This allows the user to intuitively zoom into the geographical area of interest.

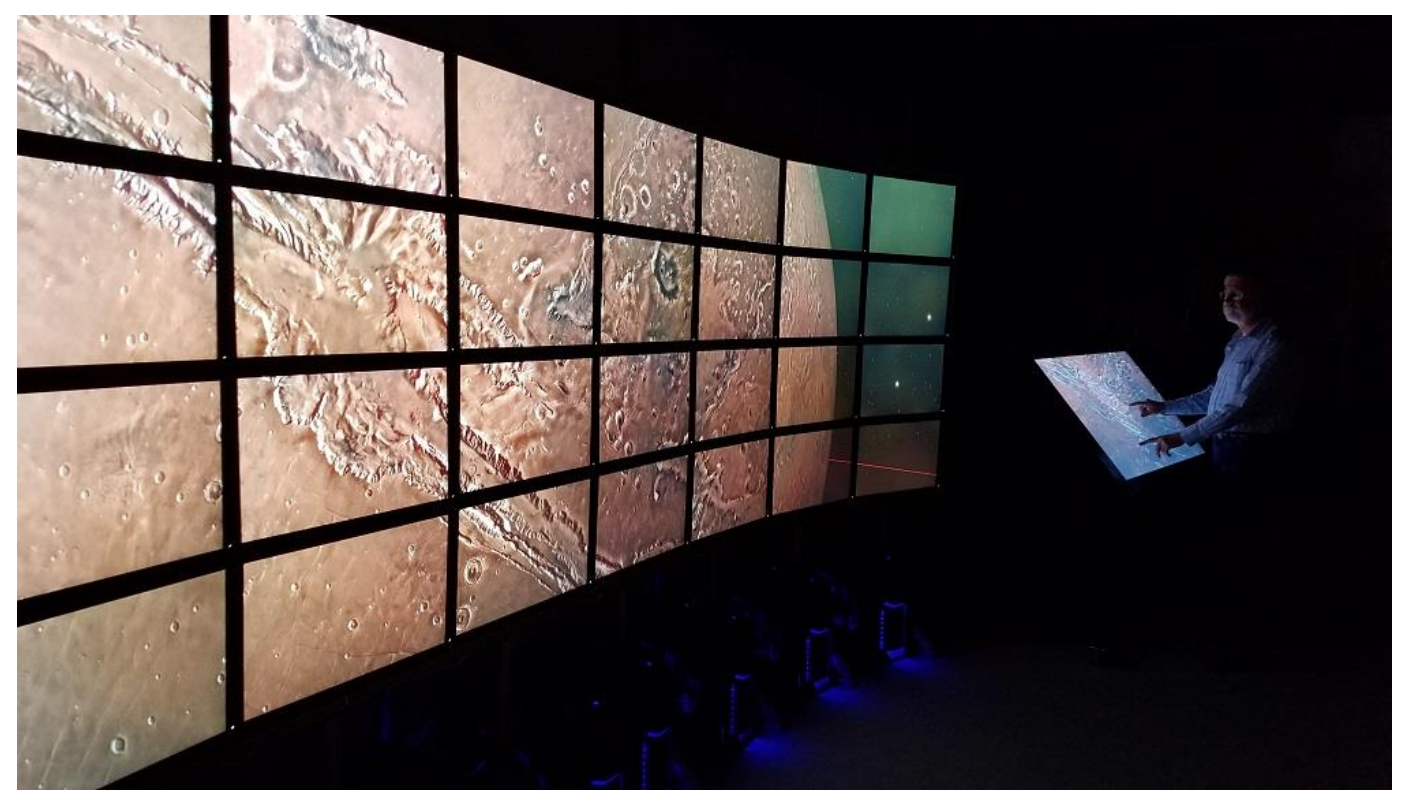

Figure 2. Multi-Touch table driving a power-wall using a linked OpenSpace session such that the viewpoint on the power-wall is controlled by the touch-table. The imagery is the Valles Marineris of Mars obtained from satellites orbiting Mars and streamed to OpenSpace through WMS.

Additionally, OpenSpace provide the capability for coupling OpenSpace sessions across devices. These could be multiple planetariums, as was done for the New Horizons encounter coupling ANMH/Hayden Planetarium with the Norrköping Visualiseringscenter C Planetarium for a cross-continent, interactive visualization and story-telling of the mission as the encounter was taking place. With the multi-touch interface, we have found it advantageous to couple the touch-table interface with a large tiled display to effectively contextualize NASA missions to groups of K-12 students and the general public. Figure 2 shows the table driving the tiled display wall to seamlessly control the OpenSpace session for multiple participants. A YouTube video of the interaction is available?

\section{OSIRIS-REx Mission}

OSIRIS-REx launched on September 8, 2016 at 7:05 PM EDT from Cape Canaveral. Figure 3 shows the trail of the spacecraft a few minutes after lift-off from Cape Canaveral. OSIRIS-REx orbited the Sun and in September 2017, it used a gravity assist in an Earth fly-by to increase the orbital inclination to deviate from an orbit in the solar plane to an orbit matching the orbital plane of asteroid Bennu. The "slingshot" to the orbital plane of Bennu is shown in Figure 4. 


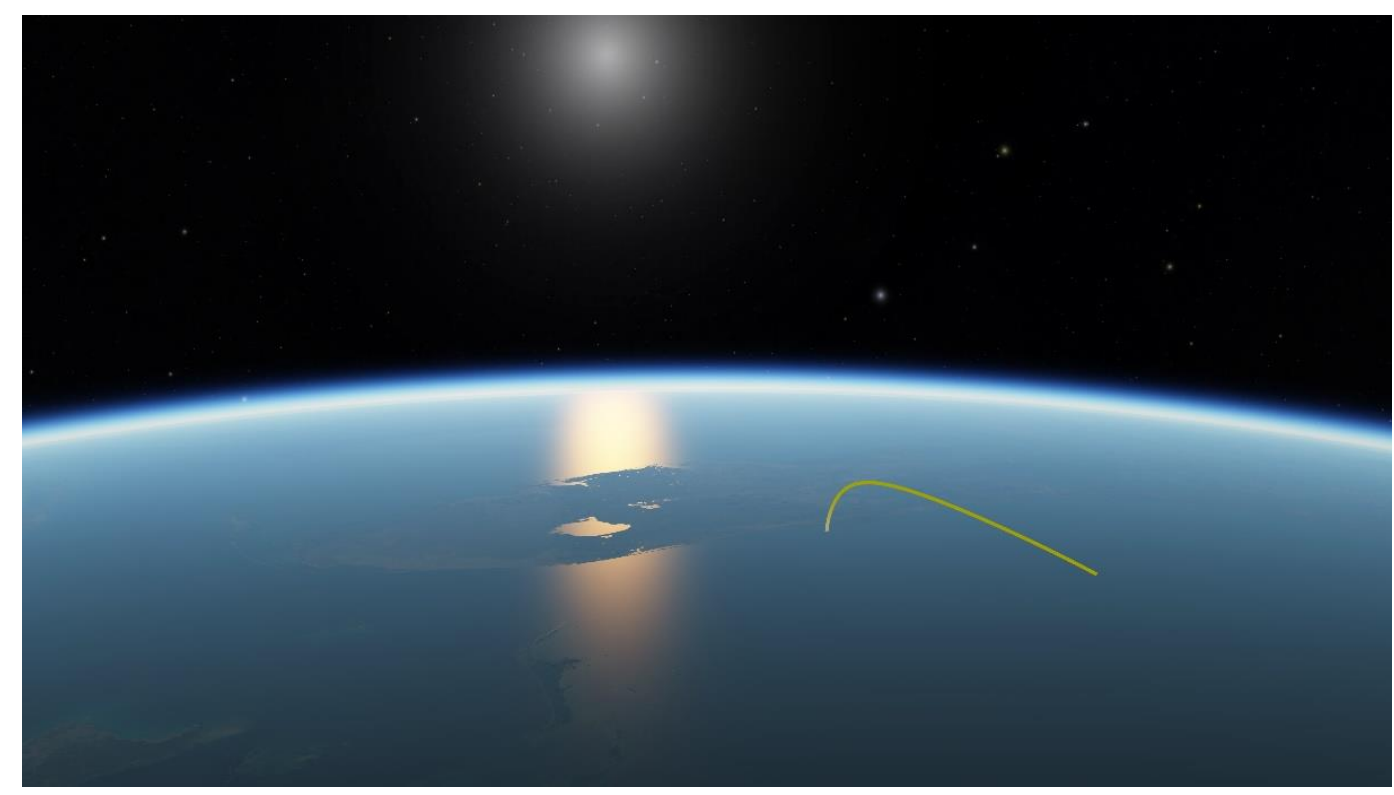

Figure 3. OSIRIS-REx lift-off from Cape Canaveral on September 8, 2016. The location of the spacecraft at a particular time-point is determined from the SPICE data for this mission.

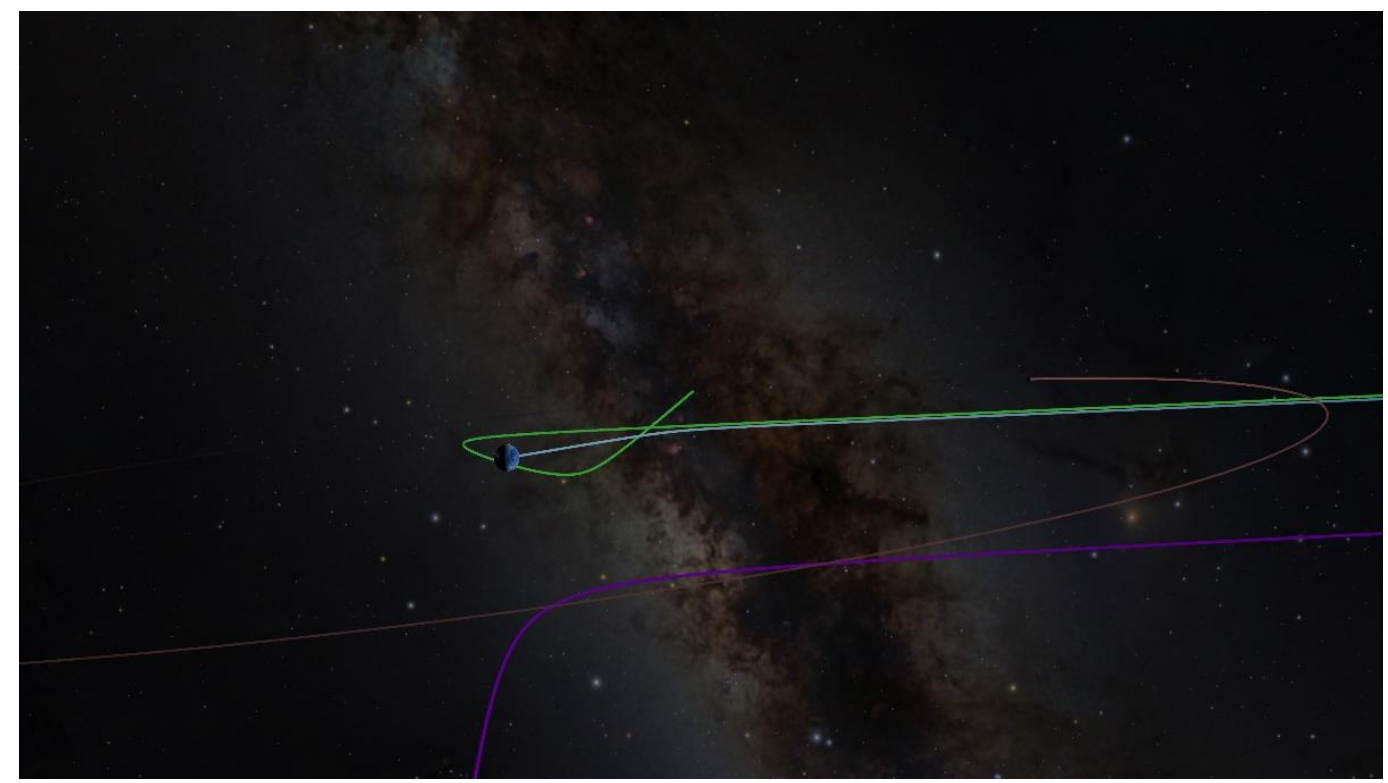

Figure 4. September 22, 2017. OSIRIS-REx uses Earth's gravitational field to slingshot from the solar orbit to the orbital plane of the asteroid Bennu. Earth is the cyan trail while OSIRIS-REx is the green trail. The celestial bodies and spacecraft locations are read from SPICE data for the OSIRISRex mission.

The OSISRIS-REx mission seeks to gather a sample from the surface of Bennu, a carbonaceous asteroid and return the sample to Earth. The sample may provide information on the formation of the life on Earth and the Earth's oceans. Bennu may contain organics, precious metals, and water. The sample should provide information on the contents. In addition to the sample, the mission will map the asteroid and use its advanced instruments to measure the Yarkovsky effect (non-gravitational forces which cause orbit deviation) as well as comparing the close-range observations with Earth-based observations. 
By using NASA's SPICE kernels for this mission, the reconnaissance campaign mapping the asteroid can be visualized and the instrument activation highlighted. This is shown in Figure 5. By using the actual mission planning data contained in the NASA SPICE event file which contains the timeline of the mission, a visualization and contextualization of the mission is accomplished. This can be shown in an immersive environment to describe the mission to the public and inform them of the scientific actions taken by the mission to better inform and educate the public.

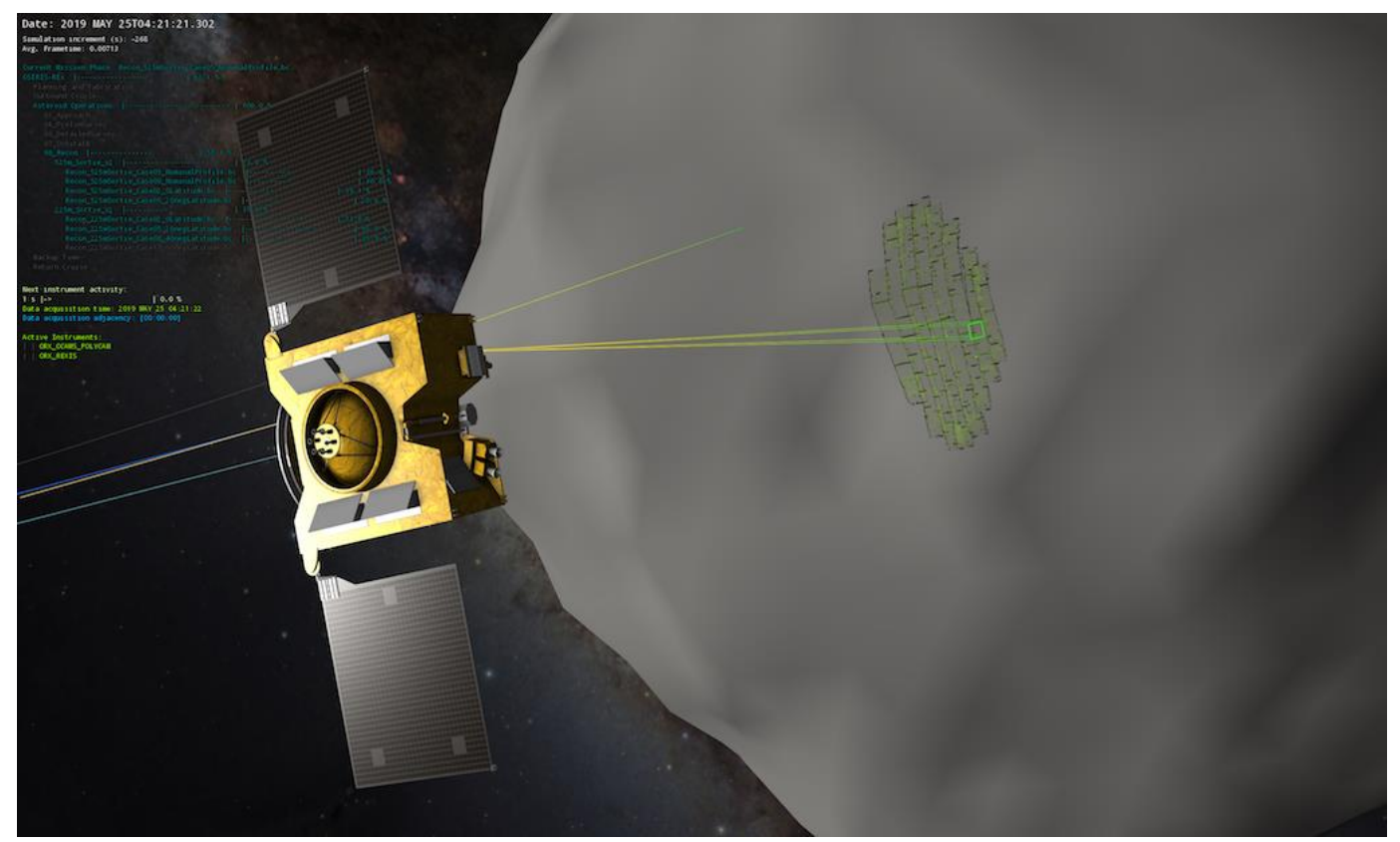

Figure 5. May 25, 2019. OSIRIS-REx is mapping Bennu before the samples are obtained. The spacecraft and Bennu are modeled as geometry. Once the actual images are sent back to Earth, the mapping would appear as textures on the asteroid surface. The spatial/temporal locations of the asteroid, spacecraft and its instruments are read from the SPICE data for this mission.

\section{CONCLUSION}

We have described OpenSpace, a software for the visualization and demonstration of NASA missions. The ability to interactively display and communicate missions during mission planning and/or mission activity and/or post-mission data analysis is a powerful method for public education. One key component is the ability to visualize the data acquisition operations from the viewpoint of the spacecraft using SPICE data. Immersive environments add to the realism of presenting NASA missions and a variety of immersive devices are supported; from display walls to touch-tables to multi-user immersive theaters such as planetariums. We believe extensions to educate the public on space science, such as explaining the importance of space weather, will result in better public awareness and support of NASA missions. Our viewpoint is that diverse information needs to be aggregated and made available to visualization software that can integrate the multiple sources allowing a coherent visual communication to a wide variety of audiences and space science consumers. This requires the flexibility of a number of delivery and interaction mechanisms from the software. 


\section{REFERENCES}

1. Analytical Graphics, Inc. The systems toolkit. www.agi.com/products/stk

2. S. Klashed, P. Hemingsson, C. Emmartand, M. Cooper, and A. Ynnerman, "Uniview Visualizing the Universe" Eurographics 2010 - Areas Papers, pp. 37-43, 2010.

3. A. Bock, M. Marcinkowski, J. Kilby, C. Emmart, and A. Ynnerman, "OpenSpace: Public dissemination of space mission profiles" IEEE VIS Posters, pp. 141-142, 2015.

4. E. Axelsson, J. Costa, C. Silva, C. Emmart, A. Bock, and A. Ynnerman, "Dynamic Scene Graph: Enabling Scaling, Positioning, and Navigation in the Universe," Computer Graphics Forum, Vol. 36, No. 3, pp. 459-468, June 2017.

5. K. Bladin, E. Axelsson, E. Broberg, C. Emmart, P. Ljung,

A. Bock, and A. Ynnerman, "Globe Browsing: Contextualized Spatio-Temporal Planetary Surface Visualization," IEEE Transactions on Visualization and Computer Graphics, Vol. 24, No. 1, pp. 802-811, January 2018.

6. C. Emmart, A. Ynnerman, A. Bock, M. Kuznetsova, R. Kinzler, V. Trakinski, M. Mac Low, D. Ebel, "OpenSpace: From Data Visualization Research to Planetariums and Classrooms Worldwide.”, American Geophysical Union, Fall General Assembly 2016, abstract.

7. Tobias Isenberg, "Interactive Exploration of Three-Dimensional Scientific Visualizations on Large Display Surfaces", Collaboration Meets Interactive Spaces, Springer, 2016, pp. 97-12.

8. Interspectral $\mathrm{AB}$, Commercial provider of touch table technology: www.interspectral.com.

9. Jonathan Bosson, "Touchtable Direct-manipulation with OpenSpace" https://youtu.be/uL-2Rpdl-68, Jul 13, 2017

10. A. Bock, E. Axelsson, C. Emmart, M. Kuznetsova, C. Hansen, A. Ynnerman, "OpenSpace -- Changing the narrative of public dissemination in astronomical visualization from What to How", IEEE Computer Graphics and Applications, Special Issue - Applied Vis, Vol. 38, No. 3, to appear May/June 2018

\section{AUTHOR BIOS}

Alexander Bock is a Moore-Sloan Postdoctoral Research fellow with New York University's Center for Data Science. Contact him at alexander.bock@nyu.edu.

Charles Hansen is a Professor of Computer Science in the School of Computing and an Associate Director of the Scientific Computing and Imaging Institute at the University of Utah. Contact him at hansen@ sci.utah.edu.

Anders Ynnerman is a Professor of Scientific Visualization at Linköping University, Sweden and an Adjunct Professor at the University of Utah. He is also the director of the Norrköping Visualization center and one of the co-founders of the OpenSpace project. Contact him at anders.ynnerman@liu.se.

Contact department editor Theresa-Marie Rhyne at theresamarierhyne@ gmail.com. 